\title{
Toppling dynamics of a mass-varying domino system
}

\author{
Tengfei Shi · Yang Liu - Nannan Wang - Caishan Liu
}

Received: date / Accepted: date

\begin{abstract}
This paper studies the toppling dynamics of a mass-varying domino system for which the mass of the domino changes at an exponential rate of its sequence number. By introducing geometrical constraints representing the interactions between the dominoes and the ground, we propose a simplified model which can describe their toppling dynamics as a one-degree-of-freedom structural-varying system. Principles of energy and generalized momentum conservation are used to investigate the free falling and the colliding phases of the system, and our proposed model is validated through a comprehensive numerical study. Furthermore, we develop an impact mapping for studying the evolution of the system by using the mathematical properties of their geometrical constraints, and establish the occurrence conditions for solitary wave. According to these conditions, the system can exhibit four different propagation modes: non-solitary mode, uniform solitary mode, accelerating solitary mode and stopped mode. Based on the studies in this paper, we can reveal the dynamic characteristics of domino phenomenon and provide an insight into its energy evolution.
\end{abstract}

Keywords Domino system · Domino phenomenon · Rigid body dynamics - Wave propagation

T. Shi $\cdot$ N. Wang $\cdot$ C. Liu*

State Key Laboratory for Turbulence and Complex Systems, College of Engineering, Peking University, Beijing, China, 100871

E-mail: liucs@pku.edu.cn

Y. Liu

College of Engineering, Mathematics and Physical Sciences, University of Exeter, North Park Road, Exeter, UK, EX4 $4 \mathrm{QF}$

\section{Nomenclature}

d Thickness of domino (m)

$e \quad$ Coefficient of restitution (-)

E Mechanical energy (J)

$F^{n} \quad$ Normal contact force $(\mathrm{N})$

$F \tau \quad$ Tangential contact force (N)

$\mathcal{F}_{I} \quad$ Inertial coordinate frame (-)

$\mathcal{F}_{i} \quad$ Body fixed coordinate frame (-)

$g \quad$ Gravitational acceleration $\left(\mathrm{m} / \mathrm{s}^{2}\right)$

$h \quad$ Hight of domino (m)

$\mathbf{i}, \mathbf{j}, \mathbf{i}_{i}, \mathbf{j}_{i} \quad$ Unit vectors in coordinate systems (-)

$i, j, n \quad$ Sequence index (-)

$I^{c} \quad$ Moment inertia about mass centre $\left(\mathrm{kg} \cdot \mathrm{m}^{2}\right)$

I Moment inertia about lower right corner $\left(\mathrm{kg} \cdot \mathrm{m}^{2}\right)$

$\lambda \quad$ Number of the discrete points on contact interface (-)

$k \quad$ Attenuation exponent of domino's motion (-)

$m_{i} \quad$ Mass of $i$ th domino $(\mathrm{kg})$

$q \quad$ Mass exponent (-)

q Column vector of generalized coordinates (-)

$s \quad$ Spacing between dominoes (m)

$t \quad$ Time $(\mathrm{s})$

$T \quad$ Kinetic energy $(\mathrm{J})$

$u \quad$ Dimensionless height of the center of mass (-)

$v^{\tau} \quad$ Relative tangential velocity $(\mathrm{m} / \mathrm{s})$

$V \quad$ Gravitational potential energy $(\mathrm{J})$

$x, y \quad$ Position of domino's centre (m)

$\theta_{i} \quad$ Rotation angle of $i$ th domino (rad)

$\theta_{c} \quad$ Domino's spacing angle (rad)

$\theta_{m} \quad$ Domino's maximum tilt angle (rad)

$\delta \quad$ Relative normal displacement (m)

$\mu \quad$ Slip friction coefficient (-)

$\mu^{s} \quad$ Stick friction coefficient (-)

$\Psi_{n}^{i} \quad$ Angle of $i$ th domino when the $n$th domino's angle is $\theta$ (rad)

$\Phi_{n}^{i} \quad$ Ratio of the angular velocities between the $i$ th and the $n$th dominoes (-) 


\section{Introduction}

Domino phenomenon is usually used to describe the cumulative effect in a marginally stable system when one event sets off a chain of similar events, such as the wave propagation in earthquake [1, 2], the transitions within a cell cycle [3], the collapsing of carbon nanotube [4, 5], the ruck in a rug [6, 7], nuclear and chemical reactions [8, 9], energy transmission in meta-material 10-14, and the chain effect in economics and sociology [15, 16]. These phenomena can be physically represented by the domino falling problem [17], i.e., a group of regularly spaced dominoes fall down one by one if an initial push is given leading to a domino wave. In particular, the propagation speed and the energy evolution of the domino wave are the main characteristics studied by many researchers for understanding different chain events, e.g. [18 28].

Modelling the dynamics of dominoes has attracted great attention from many researchers. Daykin [18] firstly pioneered the problem of domino toppling, and Shaw [19] studied the toppling domino chain experimentally. McLachlan et al. [20] proposed that the domino wave can propagate at a constant front speed depending on the spacing between two neighboring dominoes. Bert 21] studied the free toppling of a single domino, and found an explicit relationship for its front speed and domino's spacing. In [22], Efthimiou et al. deduced the front speed for a domino array by assuming the domino as a massless rod with a concentrated mass at its top. Larham [23] conducted extensive experiments and found that the model proposed by Efthimiou et al. 22] is rather limited. In order to analyze domino's propagation, Stronge developed two types of models, a single collision model 24] and a cooperative group model [25]. By comparing both models numerically and experimentally, it shows that the single collision model can be applied to the dominoes with a large spacing, while the cooperative group model, which assumes frictionless and perfectly plastic contact between each domino, can predict the trend of domino wave for any spacing. van Leeuwen [26] and Fujii et al. 27] further developed the cooperative group model by taking friction into account. Furthermore, Lu et al. [28] applied the discrete element method to simulate domino's falling, and Shi et al. 17] developed a precise numerical model with consideration of multipoint impacts between dominoes. Until now, most of the existing studies have focused on studying the characteristics of the domino wave by using uniform dominoes, and the studies of nonuniform dominoes are very limited. Therefore, this paper will study the domino wave by extending the precise numerical model proposed in [17] and the cooperative group model studied in [25] to nonuniform dominoes.

In this paper, we consider a special nonuniform domino array, namely the mass-varying domino system, for which both the mass and the inertia of the domino exponentially change with its sequence number, while the size and spacing of the dominoes are uniform. To study the toppling dynamics of the domino system, we will develop a numerical model, for which the algorithm for multiple impacts [29, 30] will be introduced to the impact process of the dominoes, and the linear complementary conditions [31] will be applied to its contact dynamics. We will also develop a simplified model for the nonuniform dominoes by following the assumptions of the cooperative group model proposed in [25]. This simplified model assumes that the interaction between the domino and the ground satisfies some geometrical constraints, and therefore, the dominoes can be described as a onedegree-of-freedom structure-varying system. Based on the simplified model, the impact-free dynamics of the system is governed by the conservation principle of mechanical energy, while the collision at the wave front satisfies the principle of the generalized momentum conservation. Furthermore, we will extensively analyse the evolution of the kinetic energy and the gravitational potential energy based on the mathematical properties of geometrical constraints of the domino system. Then we will establish the impact mapping to reveal the evolution of domino's front speed, as well as the condition for which allows the domino wave to propagate in a form of solitary wave. Finally, according to various combinations of domino's mass and geometric parameters, our theoretical results indicate four types of propagation modes for the domino wave: non-solitary mode, uniform solitary mode, accelerating solitary mode and stopped mode.

The rest of this paper is organised as follows. Numerical and the simplified models of nonuniform dominoes are introduced in Section 2 and 3. respectively. In Section 4, some analytical solutions of domino's propagation are presented. In Section 5. numerical calculations are carried out to validate the accuracy of the simplified model. In addition, the dependence of wave's characteristics, such as propagation mode, front speed and energy evolution, on the geometry and mass variation of the domino system are studied numerically. Finally, some conclusions are drawn in Section 6 . 


\section{Numerical model}

The falling dominoes considered in this study are shown in Fig. 1(a), where $h$ and $d$ are the height and the thickness of the domino, respectively, and $s$ is the spacing between two neighboring dominoes. Let $m_{i}$ be the mass of the $i$ th domino, the moment of inertia of the $i$ th domino about its centre of mass can be written as $I_{i}^{c}=m_{i}\left(h^{2}+d^{2}\right) / 12$.

Define an inertial coordinate frame $\mathcal{F}_{I}=\{O ; \mathbf{i j}\}$ with the axial $\mathbf{i}$ and $\mathbf{j}$ along and perpendicular to the ground, respectively. A body coordinate $\mathcal{F}_{i}=\left\{O_{i} ; \mathbf{i}_{i} \mathbf{j}_{i}\right\}$ fixed at the centre of mass of the $i$ th domino $O_{i}$ with the axial $\mathbf{i}_{i}$ and $\mathbf{j}_{i}$ perpendicular and along the domino, respectively. The motion of the $i$ th domino can be described by a generalized coordinate $\mathbf{q}_{i}=\left(x_{i}, y_{i}, \theta_{i}\right)$, where $\left(x_{i}, y_{i}\right)$ is the position of the $i$ th domino's centre $O_{i}, \theta_{i}$ is its angular displacement, and $\theta_{i}>0$ means the clockwise rotation of the $i$ th domino.

If the $(n-1)$ th domino is falling down towards its stable neighbor $n$, the generalized coordinates for the current configuration of the system can be written as $\mathbf{q}=\left(\mathbf{q}_{1}, \mathbf{q}_{2}, \cdots, \mathbf{q}_{n}\right)^{T} \in \mathbb{R}^{3 n}$, which includes the fallen dominoes only, and its dimension varies with the number of dominoes that are falling.

For the first (n-1) dominoes, we define the pointcontact pair between the $i$ th domino and the ground as $\left\{A_{i}, A_{i}^{\prime}\right\}$, and the point-contact pair between the $i$ th and the $(i+1)$ th dominoes as $\left\{B_{i}, B_{i}^{\prime}\right\}$. For the $n$th domino, line contact between the domino and the ground is considered, which can be simplified as a series of discrete point contacts $\left\{A_{n j}, A_{n j}^{\prime}\right\}$ as shown in Fig. 1(b), where $j=1,2, \cdots, \lambda$, and $\lambda$ is the number of these discrete points to be defined. Therefore, the total number of possible contact points in this system is $n_{c}=$ $2 n-1+\lambda$.

For $n$ falling dominoes, the relative normal displacements of these contact pairs can be written as

$\boldsymbol{\delta}=\left[\delta_{A_{1}}, \delta_{B_{1}}, \cdots, \delta_{A_{n-1}}, \delta_{B_{n-1}}, \delta_{A_{n 1}}, \cdots, \delta_{A_{n \lambda}}\right]^{T}$,

where $\boldsymbol{\delta} \in \mathbb{R}^{n_{c}}, \delta_{A_{i}}$ and $\delta_{B_{i}}$ are the relative normal displacements of the contact pairs $\left\{A_{i}, A_{i}^{\prime}\right\}$ and $\left\{B_{i}, B_{i}^{\prime}\right\}$, respectively, and $\delta_{A_{n j}}$ is the relative normal displacement of the contact pair $\left\{A_{n j}, A_{n j}^{\prime}\right\}$. The contact forces between these contact points can be decomposed along the normal and the tangential directions as

$\left\{\begin{array}{l}\mathbf{F}^{n}=\left[F_{A_{1}}^{n}, F_{B_{1}}^{n}, \cdots, F_{A_{n-1}}^{n}, F_{B_{n-1}}^{n}, F_{A_{n 1}}^{n}, \cdots, F_{A_{n \lambda}}^{n}\right]^{T} \\ \mathbf{F}^{\tau}=\left[F_{A_{1}}^{\tau}, F_{B_{1}}^{\tau}, \cdots, F_{A_{n-1}}^{\tau}, F_{B_{n-1}}^{\tau}, F_{A_{n 1}}^{\tau}, \cdots, F_{A_{n \lambda}}^{\tau}\right]^{T}\end{array}\right.$ where $F_{i}^{n} \in \mathbb{R}^{n_{c}}$ and $F_{i}^{\tau} \in \mathbb{R}^{n_{c}}$ are the normal and tangential contact forces of the $i$ th contact point, respectively.

By using the Euler-Lagrange equation, we can obtain the equation of motion for the domino system as

$\mathbf{M} \ddot{\mathbf{q}}=\mathbf{G}+\mathbf{W F}^{n}+\mathbf{N F}^{\tau}$,

where $\mathbf{M}=\operatorname{diag}\left(m_{1}, m_{1}, I_{1}^{c}, m_{2}, m_{2}, I_{2}^{c}, \cdots, m_{n}, m_{n}\right.$, $\left.I_{n}^{c}\right) \in \mathbb{R}^{3 n \times 3 n}$ is the generalized mass matrix, $\mathbf{G}=\left[0,-m_{1} g, 0,0,-m_{2} g, 0, \cdots, 0,-m_{n} g, 0\right]^{T} \in \mathbb{R}^{3 n \times 1}$ is the generalized gravity, $g$ is the gravitational acceleration, $\mathbf{W} \in \mathbb{R}^{3 n \times n_{c}}$ and $\mathbf{N} \in \mathbb{R}^{3 n \times n_{c}}$ are the Jacobian matrices for connecting the normal and tangential forces to the generalized forces, respectively. A detailed derivation of these matrices and the solution of Eq (3) can be found from 17].

To solve Eq. (3), we divide the process of domino falling into an impact-free and an impact phases. For the impact-free phase, the normal contact force $F_{i}^{n}$ can be obtained by using the integrated linear complementary relationship established in the displacement, velocity and acceleration [32, 33], which can be written as

$\left\{\begin{array}{l}\delta_{i} \cdot F_{i}^{n}=0, \quad \delta_{i} \geq 0, \quad F_{i}^{n} \geq 0, \\ \dot{\delta}_{i} \cdot F_{i}^{n}=0, \quad \dot{\delta}_{i} \geq 0, \quad F_{i}^{n} \geq 0, \\ \ddot{\delta}_{i} \cdot F_{i}^{n}=0, \quad \ddot{\delta}_{i} \geq 0, \quad F_{i}^{n} \geq 0,\end{array}\right.$

where $i=1,2, \cdots, n_{c}$. To determine the tangential contact force $F_{i}^{\tau}$, Coulomb friction is introduced as below

$F_{i}^{\tau}= \begin{cases}F_{i}^{\tau}=-\mu_{i} F_{i}^{n} \frac{v_{i}^{\tau}}{\left|v_{i}^{\tau}\right|}, & \text { if } v_{i}^{\tau} \neq 0, \\ F_{i}^{\tau} \leq \mu_{i}^{s} F_{i}^{n}, & \text { if } v_{i}^{\tau}=0,\end{cases}$

where $\mu_{i}>0$ and $\mu_{i}^{s}>\mu_{i}$ are the slip and the stick friction coefficients at the $i$ th contact set, respectively. Based on the above conditions, they can be used for solving Eq. (3) in the impact-free phase.

Once an impact occurs, the interaction at the contact point for short time duration may cause stiff problem in numerical computation 34. To avoid this issue, we employ the multi-point impact model proposed in 29, 30, 32 by neglecting the non-impulsive forces (e.g. gravity), and assume invariant displacement during the impact. Under these two assumptions, Eq. (3) can be transform into an impulse equation for the impact phase of the domino system as

$\mathbf{M d} \dot{\mathbf{q}}=\mathbf{W} \mathrm{d} \mathbf{P}^{n}+\mathbf{N d} \mathbf{P}^{\tau}$,

where $\mathrm{d} \mathbf{P}^{n}=\boldsymbol{F}^{n} \mathrm{~d} t$ and $\mathrm{d} \mathbf{P}^{\tau}=\mathbf{F}^{\tau} \mathrm{d} t$ are the normal and the tangential infinitesimal impulses, respectively. To solve Eq. (6), $\mathrm{d} \mathbf{P}^{n}$ and $\mathrm{d} \mathbf{P}^{\tau}$ should be obtained 


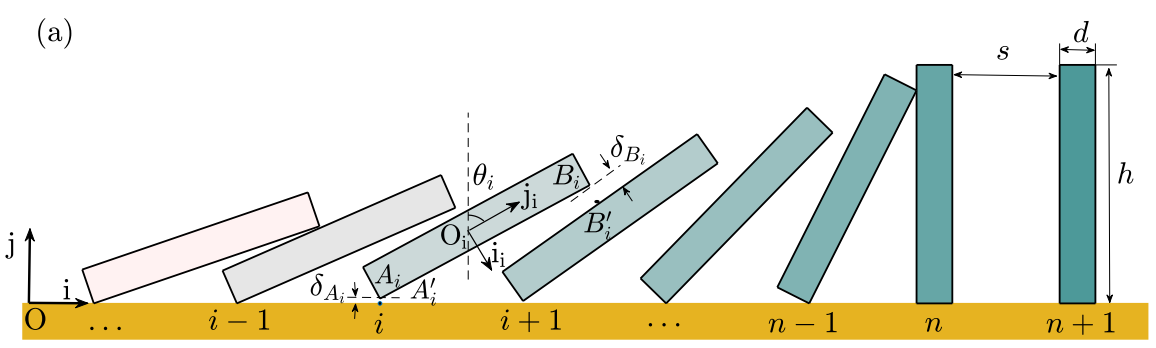

(b)

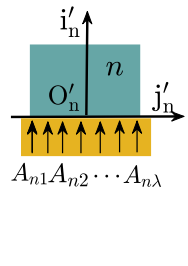

Fig. 1 (a) Toppling dominoes on the ground. (b) Discrete contact points model.

through the distributing rule of impulse [29, 30], which determines the ratios of different infinitesimal impulses at various contact points. The ratio in the distributing rule can be determined through the stiffness of the contact points and the potential energy stored in the contact sets, while the stiffness can be described by introducing a constitution law between the relative normal displacement and the normal contact force at the contact point. For this reason, the Hertz contact model 35] was adopted in this study. On the other hand, the potential energy between contact sets can be induced by the work done by the normal contact force through normal deformation, and this quantity can also be obtained by multiplying the relative normal velocity and impulse. Here, it is worth noting that, due to the plastic impact in the system, the bilinear constitutive model 36] was used for calculating the energy dissipation in this process, and the tangential infinitesimal impulses was calculated through the normal infinitesimal impulses using the Coulomb friction law. For a detailed study of this impact phase, readers can refer to [32].

\section{Simplified model}

\subsection{Motion of dominoes}

Numerical studies in [17] have shown that the physical parameters of dominoes, such as friction and restitution coefficients, have less influence than their geometrical parameters, e.g. thickness and spacing, on the toppling dynamics of the dominoes. Following the assumptions of the cooperative group model in [25], we restrict the interactions between the dominoes and the ground in our system as follows.

(a) There is no sliding motion occurring between the domino and the ground;

(b) The impact between the ground and the domino is perfectly plastic, i.e. the domino is kept in contact with the ground after the impact;

(c) The friction between dominoes is neglectable; (d) The impact between two dominoes is perfectly plastic, i.e. two colliding dominoes remain in contact after the impact.

Fig. 2 shows the snapshot of a toppling domino system under the above assumptions. As shown in this picture, the relative motion between two neighboring dominoes satisfies the following geometric constraint [25]

$$
\begin{aligned}
\theta_{i}(t) & =\theta_{i+1}(t)+\arcsin \left[\frac{1}{h}\left((s+d) \cos \theta_{i+1}(t)-d\right)\right] \\
& \triangleq \psi\left(\theta_{i+1}(t)\right),
\end{aligned}
$$

where $\theta_{i}(t)$ and $\theta_{i+1}(t)$ are the rotational angles of the $i$ th and the $(i+1)$ th dominoes, respectively, and here the $n$th domino is defined as the front domino. Due to the geometry of the domino, the rotational angle for each tilted domino is limited within the range $\theta_{i}(t) \in$ $\left[0, \theta_{m}\right)$, where $\theta_{m}=\arccos (d /(d+s))$, corresponding to a maximum tilt angle.

Differentiating Eq.(7) with respect to time, we obtain the relationship of the angular velocities between two neighboring dominoes,

$$
\begin{aligned}
\frac{\dot{\theta}_{i}(t)}{\dot{\theta}_{i+1}(t)} & =1-\frac{(s+d) \sin \theta_{i+1}(t)}{h \cos \left(\theta_{i}(t)-\theta_{i+1}(t)\right)} \\
& \triangleq \phi_{i+1}^{i}\left(\theta_{i+1}(t)\right) .
\end{aligned}
$$

It is clear that Eqs.(7) and (8) establish two recursive relationships that allow the motion state of each tilted domino to be determined by a single independent variable. Here, we choose the independent variable as the rotational angle of the front domino, $\theta_{s}$. According to Eqs. (7) and (8), the rotational angle $\theta_{i}$ and the angular velocity $\dot{\theta}_{i}$ of the $i$ th domino $(i \leq n)$ can be expressed as

$$
\left\{\begin{array}{l}
\theta_{i}=\Psi_{n}^{i}\left(\theta_{s}\right), \text { where } \Psi_{n}^{i}\left(\theta_{s}\right)=\underbrace{\psi\left(\psi\left(\cdots \psi\left(\theta_{s}\right)\right)\right)}_{n-i}, \\
\dot{\theta}_{i}=\Phi_{n}^{i}\left(\theta_{s}\right) \dot{\theta}_{n}, \text { where } \Phi_{n}^{i}\left(\theta_{s}\right)=\prod_{i=j}^{n-1} \phi_{j+1}^{j}\left(\theta_{s}\right),
\end{array}\right.
$$

where function $\Psi_{n}^{i}\left(\theta_{s}\right)$ represents the rotation angle of the $i$ th domino at the configuration where the front domino (the $n$th domino) has a rotational angle $\theta_{s}$, and $\Phi_{n}^{i}\left(\theta_{s}\right)$ represents the ratio of the angular velocities 


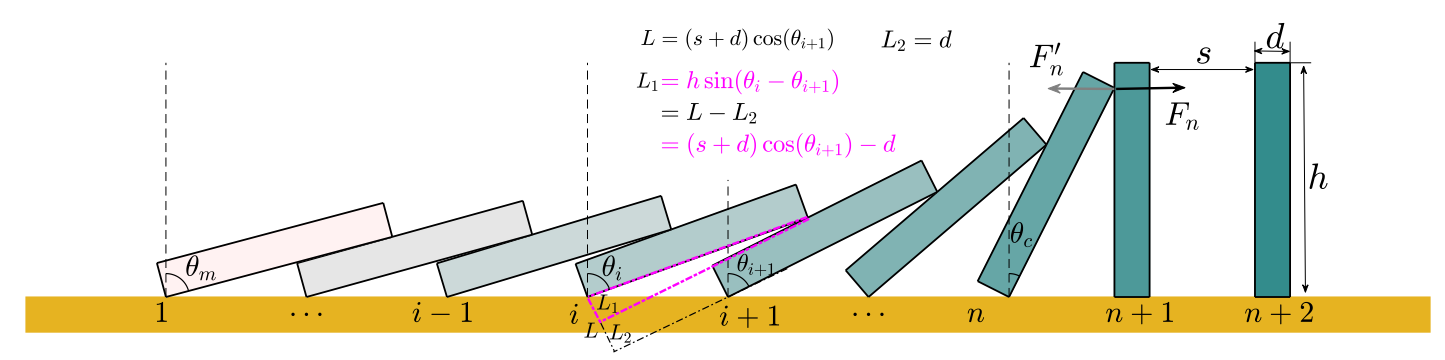

Fig. 2 A snapshot of toppling dominoes in an ideal case. The expression with red (black) colour is the geometrical constraint of the red (black) triangle between neighboring dominoes.

between the $i$ th and the $n$th dominoes. It should be noted that both functions $\Psi_{n}^{i}\left(\theta_{s}\right)$ and $\Phi_{n}^{i}\left(\theta_{s}\right)$ are dependent of the current configuration of the domino system, and $\theta_{s} \in\left[0, \theta_{c}\right]$, where $\theta_{c}=\arcsin (s / h)$, denoted as a spacing angle. According to the definitions of $\Psi_{n}^{i}\left(\theta_{s}\right)$ and $\Phi_{n}^{i}\left(\theta_{s}\right)$, we have the following properties,

$$
\left\{\begin{array}{l}
\Psi_{n}^{n}\left(\theta_{s}\right)=\theta_{s}, \\
\Phi_{n}^{n}\left(\theta_{s}\right)=1 \\
\Psi_{n}^{i}\left(\theta_{s}\right)=\Psi_{n+j}^{i+j}\left(\theta_{s}\right), \\
\Psi_{n}^{i}\left(\theta_{c}\right)=\Psi_{n+1}^{i}(0) .
\end{array}\right.
$$

The first two equations give the correlation of the front domino to itself. The third equation describes the translation of the domino configuration. The last equation describes the front transformation from $n$th domino to $(n+1)$ th domino.

Lemma 1: For a large $n$, where $n \gg i$, the angle and the angular velocity of the ith domino exponentially approach to $\theta_{m}$ and 0 , respectively, at the same ratio $k=1-\sqrt{s^{2}+2 s d} / h<1$ as the sequence number $i$ decreases.

Proof: Noting that $\theta_{m}=\psi\left(\theta_{m}\right)$, so $\theta_{m}$ is a fixed point of Eq. (7). Meanwhile, the derivation of Eq. (7) is

$\psi^{\prime}(\theta)=1-\frac{(s+d) \sin \theta}{\sqrt{h^{2}-[(s+d) \cos \theta-d]^{2}}}<1$

when $\theta \in\left[0, \theta_{m}\right)$. Therefore, the angle $\theta_{i}$ will converge to $\theta_{m}$ if $i \ll n$. In this case, we can use the Taylor series to expand $\psi\left(\theta_{i+1}\right)$ around $\theta_{m}$ as

$\theta_{i}=\psi\left(\theta_{i+1}\right) \approx \psi\left(\theta_{m}\right)+k\left(\theta_{i+1}-\theta_{m}\right)$,

where

$k=\left.\frac{\partial \psi(\theta)}{\partial \theta}\right|_{\theta=\theta_{m}}=1-\frac{\sqrt{s^{2}+2 s d}}{h}<1$,

providing that $s+d<h$. Since $\psi\left(\theta_{m}\right)=\theta_{m}$, Eq. (12) can be rewritten as

$\theta_{i}-\theta_{m} \approx k\left(\theta_{i+1}-\theta_{m}\right)$, which means that the angle $\theta_{i}$, as $i \ll n$, converges to $\theta_{m}$ at the exponential rate $k$ with decreasing $i$. Then, we can let $\theta_{i} \approx \theta_{m}$ and $\theta_{i+1} \approx \theta_{m}$ in Eq. (8) to obtain

$\frac{\dot{\theta}_{i}}{\dot{\theta}_{i+1}} \approx 1-\frac{\sqrt{s^{2}+2 s d}}{h}=k$.

Therefore, the angular velocity $\dot{\theta}_{i}$ approaches to 0 at the exponential rate $k$ as $i$ decreases. From Eq. (14) and (15), it is seen that $k$ represents the attenuation rate of the motion for the dominoes far behind the front domino.

It is obvious that, for the function sequences $\Psi_{n}^{i}\left(\theta_{s}\right)$ and $\Phi_{n}^{i}\left(\theta_{s}\right)$ when $n \gg i$, we have

$\left\{\begin{array}{l}\Psi_{n}^{i}\left(\theta_{s}\right) \approx \theta_{m}-c_{1}\left(\theta_{s}\right) k^{n-i} \\ \Phi_{n}^{i}\left(\theta_{s}\right) \approx c_{2}\left(\theta_{s}\right) k^{n-i},\end{array}\right.$

where $c_{1}\left(\theta_{s}\right)$ and $c_{2}\left(\theta_{s}\right)$ are coefficients independent of number $n$.

It is worth noting that the front wave of the domino propagation is always related to the collision between the front domino and its next neighbouring domino. Hence, the domino system is a structure-varying system containing an impact-free and an impact phases. Next, we will carry out a detailed study on these two phases.

\subsection{Impact-free dynamics}

Let us define the horizontal ground as the reference plane for zero potential energy of gravity. Consider the moment when the wave front is located at the $n$th domino with a tilt angle $\theta_{s}$, the kinematic energy $T_{n}\left(\theta_{s}\right)$ and the potential energy $V_{n}\left(\theta_{s}\right)$ at this configuration can be written as

$$
\left\{\begin{aligned}
T_{n}\left(\theta_{s}\right) & =\sum_{i=1}^{n} \frac{1}{2} I_{i} \dot{\theta}_{i}^{2}=\frac{1}{2} I_{1} \dot{\theta}_{n}^{2} K_{n}\left(\theta_{s}\right) \\
V_{n}\left(\theta_{s}\right) & =\sum_{i=1}^{n} \frac{1}{2} m_{i} g\left(h \cos \theta_{i}+d \sin \theta_{i}\right) \\
& =\frac{1}{2} m_{1} g h H_{n}\left(\theta_{s}\right),
\end{aligned}\right.
$$


where $I_{i}=m_{i}\left(h^{2}+d^{2}\right) / 3$, and $K_{n}$ and $H_{n}$ are defined as

$$
\left\{\begin{array}{l}
K_{n}\left(\theta_{s}\right)=\sum_{i=1}^{n} \frac{I_{i}}{I_{1}}\left(\Phi_{n}^{i}\left(\theta_{s}\right)\right)^{2} \\
H_{n}\left(\theta_{s}\right)=\sum_{i=1}^{n} \frac{m_{i}}{m_{1}} u\left(\theta_{i}\right)=\sum_{i=1}^{n} \frac{m_{i}}{m_{1}} u\left(\Psi_{n}^{i}\left(\theta_{s}\right)\right),
\end{array}\right.
$$

where, $\theta_{i}=\Psi_{n}^{i}\left(\theta_{s}\right)$, and $u\left(\theta_{i}\right)=\cos \left(\theta_{i}\right)+d \sin \left(\theta_{i}\right) / h$, corresponding to the dimensionless height of the center of mass for the $i$ th domino.

We use the Euler-Lagrange equation to derive the governing equations of the system, which are written as

$M_{n}\left(\theta_{s}\right) \ddot{\theta}_{s}+\frac{1}{2} \dot{\theta}_{s}^{2} S_{n}\left(\theta_{s}\right)=G_{n}\left(\theta_{s}\right)$,

where $M_{n}=I_{1} K_{n}\left(\theta_{s}\right), S_{n}\left(\theta_{s}\right)=\mathrm{d} M_{n}\left(\theta_{s}\right) / \mathrm{d} \theta_{s}$, and $G_{n}\left(\theta_{s}\right)=-\mathrm{d} V_{n}\left(\theta_{s}\right) / \mathrm{d} \theta_{s}$.

Once an initial condition is specified, the dynamical response of the domino system in an impact-free phase can then be obtained by solving Eq.(19).

\subsection{Front collision dynamics}

Let us define the collision between the front domino and its next neighbouring domino as the front collision. We have the following theorem to characterize the property of the front collision.

Theorem 1: The front collision satisfies the principle that the generalized momenta before and after the collision are conservative.

Proof: In order to analyse the impact dynamics, we use $\left\{F_{n}^{\prime}, F_{n}\right\}$ (where $F_{n}^{\prime}=-F_{n}$ ) to represent the normal impact forces acting on the $n$th and the $(n+1)$ th dominoes, respectively. Since the virtual displacement along the direction of the normal impact force $F_{n}^{\prime}$ is given by $\delta x_{n}=h \cos \theta_{s} \delta \theta_{s}$, we can write the virtual work of $F_{n}^{\prime}$ as

$\delta w_{n}=F_{n}^{\prime} \delta x_{n}=Q_{n} \delta \theta_{s}$,

where $Q_{n}=F_{n}^{\prime} h \cos \theta_{s}$.

According to Eqs. (19) and (20), the dynamics of the domino system subject to the external force $F_{n}^{\prime}$ can be written as

$M_{n}\left(\theta_{s}\right) \ddot{\theta}_{s}+\frac{1}{2} \dot{\theta}_{s}^{2} S_{n}\left(\theta_{s}\right)=G_{n}\left(\theta_{s}\right)+Q_{n}\left(\theta_{s}\right)$.

According to the conventional assumptions for rigid-body impact dynamics, the effects from the gravity and the inertial force on the impact response can be neglected. Furthermore, we assume that the configuration of the system does not change during the impact, i.e. $\theta_{s}=\theta_{c}$. Then, Eq.(21) can be simplified as

$M_{n}\left(\theta_{c}\right) \mathrm{d} \dot{\theta}_{s}=Q_{n}\left(\theta_{c}\right) \mathrm{d} t$.
Similarly, by ignoring the gravity, the dynamic equation for the $(n+1)$ th domino subject to the external force $F_{n}$ can be obtained as

$I_{n+1} \mathrm{~d} \dot{\theta}_{n+1}=F_{n} h \cos \theta_{s}=-Q_{n}\left(\theta_{c}\right) d t$.

Combining Eqs. (23) with (22) leads to

$M_{n}\left(\theta_{c}\right) \mathrm{d} \dot{\theta}_{s}=-I_{n+1} \mathrm{~d} \dot{\theta}_{n+1}$.

Defining the impact duration $\left[t^{-}, t^{+}\right]$, we have $\theta_{s}\left(t^{-}\right)=$ $\theta_{s}\left(t^{+}\right)=\theta_{c}, \theta_{n+1}\left(t^{-}\right)=\theta_{n+1}\left(t^{+}\right)=0, \dot{\theta}_{s}\left(t^{-}\right)=\dot{\theta}_{s}^{-}\left(\theta_{c}\right)$ and $\dot{\theta}_{n+1}\left(t^{-}\right)=0$. Let $\dot{\theta}_{s}\left(t^{+}\right)=\dot{\theta}_{s}^{+}\left(\theta_{c}\right)$ and $\dot{\theta}_{n+1}\left(t^{+}\right)=$ $\dot{\theta}_{n+1}^{+}(0)$ be the post-impact velocities of the $n$th and the $(n+1)$ th dominoes, respectively, the integral of Eq. (24) within the impact duration can be expressed as

$M_{n}\left(\theta_{c}\right)\left(\dot{\theta}_{s}^{+}\left(\theta_{c}\right)-\dot{\theta}_{s}^{-}\left(\theta_{c}\right)\right)=-I_{n+1} \dot{\theta}_{n+1}^{+}(0)$,

which can be reformatted into

$M_{n}\left(\theta_{c}\right) \dot{\theta}_{s}^{+}\left(\theta_{c}\right)+I_{n+1} \dot{\theta}_{n+1}^{+}(0)=M_{n}\left(\theta_{c}\right) \dot{\theta}_{s}^{-}\left(\theta_{c}\right)$.

Note that the post-impact velocities $\dot{\theta}_{s}^{+}\left(\theta_{c}\right)$ and $\dot{\theta}_{n+1}^{+}(0)$ satisfy the kinematic relationship given by Eq. (8), from which we have

$\frac{\dot{\theta}_{s}^{+}\left(\theta_{c}\right)}{\dot{\theta}_{n+1}^{+}(0)}=1-\frac{(s+d) \sin \theta_{n+1}\left(t^{+}\right)}{h \cos \left(\theta_{s}\left(t^{+}\right)-\theta_{n+1}\left(t^{+}\right)\right)}=1$.

In addition, since $M_{n}\left(\theta_{c}\right)+I_{n+1}=M_{n+1}(0)$, which corresponds to the generalized mass at the instant when the front collision just finishes, Eq. (26) can be rewritten as

$M_{n+1}(0) \dot{\theta}_{n+1}^{+}(0)=M_{n}\left(\theta_{c}\right) \dot{\theta}_{s}^{-}\left(\theta_{c}\right)$

which means that the generalized momenta before and after the collision are conservative.

Finally, by combining Eqs. (19) and (28), the entire evolution of domino's propagation can be calculated recursively.

\section{Analytical solution for the domino wave}

This section will firstly study the characteristics of kinetic and potential energy of the mass-varying domino system. Then, we will use the simplified model proposed in Section 3 to build an impact mapping, and therefore, the relationship between the postimpact speeds of two adjacent front collisions can be established. 
4.1 Characteristics of kinetic and potential energy

Suppose that the mass and the moment of inertia of the domino system change at the exponential rate $q$ with its sequence number. So, the mass and the moment of inertia of the $i$ th domino can be calculated as $m_{i}=m_{1} q^{i-1}$ and $I_{i}=I_{1} q^{i-1}$, respectively. For the $n$-dominoes system whose front domino is located at a configuration with an angle $\theta_{s}$, its coefficients in Eq. (18) can be calculated as

$$
\left\{\begin{aligned}
K_{n}\left(\theta_{s}\right) & =\sum_{i=1}^{n} q^{i-1}\left(\Phi_{n}^{i}\left(\theta_{s}\right)\right)^{2} \\
& =\left(\Phi_{n}^{1}\left(\theta_{s}\right)\right)^{2}+q \sum_{i=2}^{n} q^{i-2}\left(\Phi_{n}^{i}\left(\theta_{s}\right)\right)^{2} \\
H_{n}\left(\theta_{s}\right) & =\sum_{i=1}^{n} q^{i-1} u\left(\Psi_{n}^{i}\left(\theta_{s}\right)\right) \\
& =u\left(\Psi_{n}^{1}\left(\theta_{s}\right)\right)+q \sum_{i=2}^{n} q^{i-2} u\left(\Psi_{n}^{i}\left(\theta_{s}\right)\right)
\end{aligned}\right.
$$

When $\theta_{s}=0$, according to the last equation in Eq.(10), we have $\theta_{n-1}=\theta_{c}$. So, from the first equation in Eq.(29) we obtain

$$
\begin{aligned}
K_{n}(0) & =\sum_{i=1}^{n-1} q^{i-1}\left(\Phi_{n-1}^{i}\left(\theta_{c}\right)\right)^{2}+q^{n-1} \Phi_{n}^{n}(0) \\
& =K_{n-1}\left(\theta_{c}\right)+q^{n-1}
\end{aligned}
$$

According to Eq. (10), we have $\Psi_{n}^{j+1}\left(\theta_{s}\right)=\Psi_{n-1}^{j}\left(\theta_{s}\right)$ and $\Phi_{n}^{j+1}\left(\theta_{s}\right)=\Phi_{n-1}^{j}\left(\theta_{s}\right)$. Replacing the index $i$ by $j=i-1$, we can rewrite Eq. (29) as

$$
\left\{\begin{aligned}
K_{n}\left(\theta_{s}\right) & =\left(\Phi_{n}^{1}\left(\theta_{s}\right)\right)^{2}+q \sum_{j=1}^{n-1} q^{j-1}\left(\Phi_{n-1}^{j}\left(\theta_{s}\right)\right)^{2} \\
& =\left(\Phi_{n}^{1}\left(\theta_{s}\right)\right)^{2}+q K_{n-1}\left(\theta_{s}\right), \\
H_{n}\left(\theta_{s}\right) & =u\left(\Psi_{n}^{1}\left(\theta_{s}\right)\right)+q \sum_{j=1}^{n-1} q^{j-1} u\left(\Psi_{n-1}^{j}\left(\theta_{s}\right)\right) \\
& =u\left(\Psi_{n}^{1}\left(\theta_{s}\right)\right)+q H_{n-1}\left(\theta_{s}\right) .
\end{aligned}\right.
$$

Let us first analyse the mass coefficient $K_{n}\left(\theta_{s}\right)$. By using the first equation in Eq. (31), $K_{n}\left(\theta_{s}\right)$ can be computed as

$K_{n}\left(\theta_{s}\right)$

$$
=\left(\Phi_{1}^{1}\left(\theta_{s}\right)+\cdots+\frac{\left(\Phi_{i}^{1}\left(\theta_{s}\right)\right)^{2}}{q^{i-1}}+\cdots+\frac{\left(\Phi_{n}^{1}\left(\theta_{s}\right)\right)^{2}}{q^{n-1}}\right) q^{n-1}
$$

$=A_{n}\left(\theta_{s}\right) q^{n-1}$,

which corresponds to an exponent function with respect to $q$. The coefficient $A_{n}\left(\theta_{s}\right)$ takes a property as below.

Lemma 2: For $\theta_{s} \in\left[0, \theta_{c}\right], A_{n}\left(\theta_{s}\right)>1$, and if $q>k^{2}, A_{n}\left(\theta_{s}\right)$ can converge to a constant as $n$ is sufficiently large.

Proof: Since $\Phi_{1}^{1}\left(\theta_{s}\right)=1$ and all terms in the bracketed expression in Eq.(32) are greater than zero, we have $A_{n}\left(\theta_{s}\right)>1$ for $\theta_{s} \in\left[0, \theta_{c}\right]$. According to Lemma 1 , we have $\dot{\theta}_{i} / \dot{\theta}_{i+1} \approx k$ for $n \gg i$. And, from
Eq. (16), we can obtain $\Phi_{n}^{1}\left(\theta_{s}\right)=\dot{\theta}_{1} / \dot{\theta}_{n} \approx c_{2}\left(\theta_{s}\right) k^{n-1}$. Thus, the bracketed expression in Eq. (32) forms a function sequence for which each term multiplied by $k^{2} / q$ to obtain its next term, e.g. $\frac{\left(\Phi_{i}^{1}\left(\theta_{s}\right)\right)^{2}}{q^{i-1}} \frac{k^{2}}{q} \approx$ $\frac{\left(\Phi_{i+1}^{1}\left(\theta_{s}\right)\right)^{2}}{q^{i}}$ for $i \gg 1$. Obviously, if $q>k^{2}$, the sum of the function sequence in Eq. (32), $A_{n}\left(\theta_{s}\right)$, can converge to a constant for a sufficiently large $n$.

Next, let us analyse the change of coefficient $H_{n}\left(\theta_{s}\right)$ when the rotational angle of the front domino changes from $\theta_{s}$ to $\theta_{c}$. This variation can be defined as $\Delta H_{n}\left(\theta_{s}\right)=H_{n}\left(\theta_{s}\right)-H_{n}\left(\theta_{c}\right)$. According to the second equation in Eq. (31), we have

$$
\begin{aligned}
& \Delta H_{n}\left(\theta_{s}\right) \\
& =q \Delta H_{n-1}\left(\theta_{s}\right)+u\left(\Psi_{n}^{1}\left(\theta_{s}\right)\right)-u\left(\Psi_{n}^{1}\left(\theta_{c}\right)\right) .
\end{aligned}
$$

Defining $\xi_{n}\left(\theta_{s}\right)=u\left(\Psi_{n}^{1}\left(\theta_{s}\right)\right)-u\left(\Psi_{n}^{1}\left(\theta_{c}\right)\right)$, Eq. (33) can be rewritten as

$$
\begin{aligned}
& \Delta H_{n}\left(\theta_{s}\right) \\
& =\left(\xi_{1}\left(\theta_{s}\right)+\cdots+\frac{\xi_{i}\left(\theta_{s}\right)}{q^{i-1}}+\cdots+\frac{\xi_{n}\left(\theta_{s}\right)}{q^{n-1}}\right) q^{n-1} \\
& =B_{n}\left(\theta_{s}\right) q^{n-1} .
\end{aligned}
$$

Lemma 3: $B_{n}\left(\theta_{s}\right)$ will converge to a constant if $q>k$.

Proof: As $\Psi_{n}^{1}\left(\theta_{s}\right)$ and $\left.\Psi_{n}^{1}\left(\theta_{c}\right)\right)$ converge to $\theta_{m}$ if $i$ is sufficiently large, we can use the Taylor series to expand $u(\theta)$ around $\theta_{m}$ as

$\xi_{i}\left(\theta_{s}\right)=u^{\prime}\left(\theta_{m}\right)\left(\Psi_{i}^{1}\left(\theta_{s}\right)-\Psi_{i}^{1}\left(\theta_{c}\right)\right)$

where $u^{\prime}\left(\theta_{m}\right)=\left.\frac{\partial u(\theta)}{\partial \theta}\right|_{\theta=\theta_{m}}$. According to Eq. (16) , we can substitute $\Psi_{i}^{1}\left(\theta_{c}\right) \approx \theta_{m}-c_{1}\left(\theta_{c}\right) k^{i-1}$ and $\Psi_{i}^{1}\left(\theta_{s}\right) \approx$ $\theta_{m}-c_{1}\left(\theta_{s}\right) k^{i-1}$ into Eq. (35) to obtain

$\xi_{i}\left(\theta_{s}\right)=u^{\prime}\left(\theta_{m}\right)\left(c_{1}\left(\theta_{c}\right)-c_{1}\left(\theta_{s}\right)\right) k^{i-1}$,

and this leads to

$\xi_{i+1}\left(\theta_{s}\right)=k \xi_{i}\left(\theta_{s}\right)$

Then, the bracketed expression in Eq. (34) forms a function sequence for which each term multiplied by $k / q$ equals to its next term in the sequence, e.g. $\frac{\xi_{i}\left(\theta_{s}\right)}{q^{i-1}} \cdot \frac{k}{q}=$ $\frac{\xi_{i+1}\left(\theta_{s}\right)}{q^{i}}$. Obviously, if $q>k, B_{n}\left(\theta_{s}\right)$ can converge to a constant for a large $n$.

Next, let us analyse the kinetic and the potential energy stored in the domino wave. At the configuration of the front domino with an rotational angle $\theta_{s}$, the mechanical energy of the domino system is

$$
\begin{aligned}
E_{n}\left(\theta_{s}\right) & =T_{n}\left(\theta_{s}\right)+V_{n}\left(\theta_{s}\right) \\
& =\frac{1}{2} I_{1} K_{n}\left(\theta_{s}\right)\left(\dot{\theta}_{s}\right)^{2}+\frac{1}{2} m_{1} g h H_{n}\left(\theta_{s}\right),
\end{aligned}
$$


from which the following theorem can be obtained to characterize the domino wave.

Theorem 2: if $q>k$, the mass-varying domino system can form a solitary wave for which the mechanical energy of the system concentrates on a fixed number of the dominoes closely behind the front domino.

Proof: For the domino system studied in this work, we always have $k<1$. So, if $q>k$, it gives $q>k^{2}$. According to Lemma 2 and Lemma 3, when $n$ is large enough, the fallen dominoes far behind the domino front have little contributions to the mechanical energy of the system. Therefore, we have $A_{n}\left(\theta_{s}\right)=A_{n+1}\left(\theta_{s}\right)=$ $\cdots=$ constant and $B_{n}\left(\theta_{s}\right)=B_{n+1}\left(\theta_{s}\right)=\cdots=$ constant. Hence, the domino system will propagate as a solitary wave, and the mechanical energy of the system will concentrate on a fixed number of dominoes closely behind the front domino.

\subsection{Front speed and propagation mode}

Noting that the mechanical energy of the system in an impact-free phase should keep unchanged. Therefore, when the front domino changes its configuration from $\theta_{s} \in\left[0, \theta_{c}\right]$, and its motion is free of impact, we should have $E_{n}\left(\theta_{c}\right)=E_{n}(0)$, then

$m_{1} g h \Delta H_{n}(0)=I_{1}\left(K_{n}\left(\theta_{c}\right)\left(\dot{\theta}_{n}^{-}\right)^{2}-K_{n}(0)\left(\dot{\theta}_{n}^{+}\right)^{2}\right)$,

where $\dot{\theta}_{n}^{-}$is the angular velocity of the $n$th domino just before the collision with the $(n+1)$ th domino. By using Eqs. (30), (32) and (34), Eq. (39) can be rewritten as

$\left(\dot{\theta}_{n}^{-}\right)^{2}=\frac{A_{n}(0)}{q\left(A_{n+1}(0)-1\right)}\left(\dot{\theta}_{n}^{+}\right)^{2}+\frac{m_{1} g h B_{n}(0)}{I_{1} q\left(A_{n+1}(0)-1\right)}$,

in which the first and the second terms correspond to the change in the angular velocity of the $n$th domino, respectively, which is induced by the inertia of the system and the gravitational potential energy.

According to Theorem 1, the generalized momenta before and after the collision between the $n$th and the $(n+1)$ th dominoes are conservative. Considering Eqs. (28), (30), (32) and (34), it gives

$$
\begin{aligned}
\dot{\theta}_{n+1}^{+} & =\frac{M_{n}\left(\theta_{c}\right)}{M_{n+1}(0)} \dot{\theta}_{n}^{-}=\frac{K_{n}\left(\theta_{c}\right)}{K_{n+1}(0)} \dot{\theta}_{n}^{-} \\
& =\left(1-\frac{1}{A_{n+1}(0)}\right) \dot{\theta}_{n}^{-} .
\end{aligned}
$$

Then substituting Eq. (40) into (41) provides

$$
\begin{aligned}
\left(\dot{\theta}_{n+1}^{+}\right)^{2} & =\frac{A_{n}(0)\left(A_{n+1}(0)-1\right)}{q\left(A_{n+1}(0)\right)^{2}}\left(\dot{\theta}_{n}^{+}\right)^{2} \\
& +\frac{m_{1} g h B_{n}(0)\left(A_{n+1}(0)-1\right)}{I_{1} q\left(A_{n+1}(0)\right)^{2}} \\
& \equiv r_{1}\left(\dot{\theta}_{n}^{+}\right)^{2}+r_{2},
\end{aligned}
$$

which is an impact mapping for two neighboring front collisions.

When $n \gg i$ and $q>k$, both $A_{n}(0)$ and $B_{n}(0)$ are constant. If define $a=\lim _{n \rightarrow \infty} A_{n}(0)$ and $b=$ $\lim _{n \rightarrow \infty} B_{n}(0), r_{1}$ and $r_{2}$ can be calculated as

$r_{1}=\frac{1}{q}\left(1-\frac{1}{a}\right), \quad r_{2}=\frac{m_{1} g h b}{I_{1} a} r_{1}$.

It is obvious that, if $q>1-1 / a, r_{1}<1$, and the impact mapping given in Eq. (42) will converge to a fixed point,

$\dot{\theta}_{*}^{+}=\sqrt{\frac{r_{2}}{1-r_{1}}}$.

The scenario of this case is illustrated in Fig. 3(a).

Remark 1: The solution for $\dot{\theta}_{*}^{+}$can be calculated using Eq. (44) only if $q>(1-1 / a)$. When $q \leq(1-$ $1 / a), r_{1}>1$, no solution is available for $\dot{\theta}_{*}^{+}$. This is illustrated in Fig. 3 (b).

According to Eq. (40), the constant pre-impact angular velocity of the front domino can be obtained as

$\dot{\theta}_{*}^{-}=\sqrt{\frac{a}{q(a-1)}\left(\dot{\theta}_{*}^{+}\right)^{2}+\frac{m_{1} g h b}{I_{1} q(a-1)}}$.

Therefore, we can use $\dot{\theta}_{*}^{+}$and $\dot{\theta}_{*}^{-}$to compute the mean angular velocity of the front domino as $\bar{\theta}_{*}=$ $\left(\dot{\theta}_{*}^{+}+\dot{\theta}_{*}^{-}\right) / 2$, and the analytical solution of the domino speed, i.e. the front speed of the domino system, can be computed as

$v^{*}=\frac{(s+d)}{2 \theta_{c}}\left(\dot{\theta}_{*}^{+}+\dot{\theta}_{*}^{-}\right)$.

Remark 2: It is worth noting that the domino wave can pass through the chain only if the energy importing to each standing-still domino is larger than the potential energy for keeping it stable. Therefore, if the mass exponent is too large, the domino wave could be stopped by a heavy domino.

We define the stability energy for a $n$-dominoes system as $\widetilde{E}_{n}=\frac{1}{2} m_{1} g h B_{n}\left(\theta_{e}\right) q_{c}^{n-1}$, where $\theta_{e}$ is the tilt angle of the front domino when the $n$-dominoes system achieves its maximal potential energy within an impact-free phase, $q_{c}$ represents the critical mass exponent that could stop the wave propagation. For a stable domino system with a large $n\left(q_{c}>1-1 / a\right)$, 


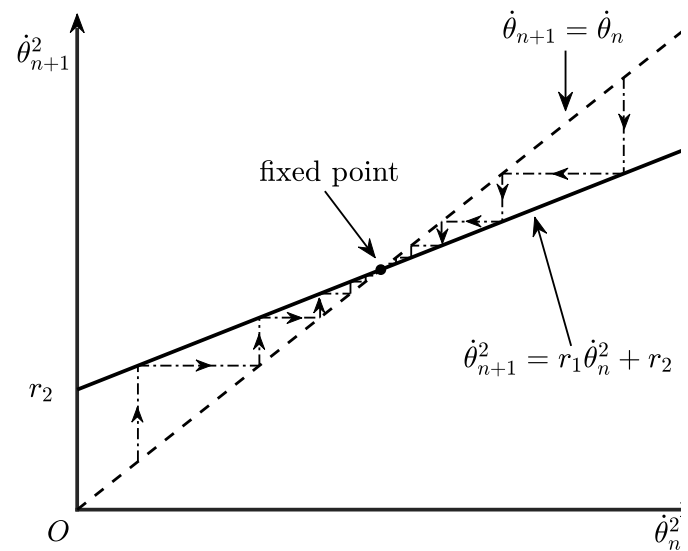

(a)

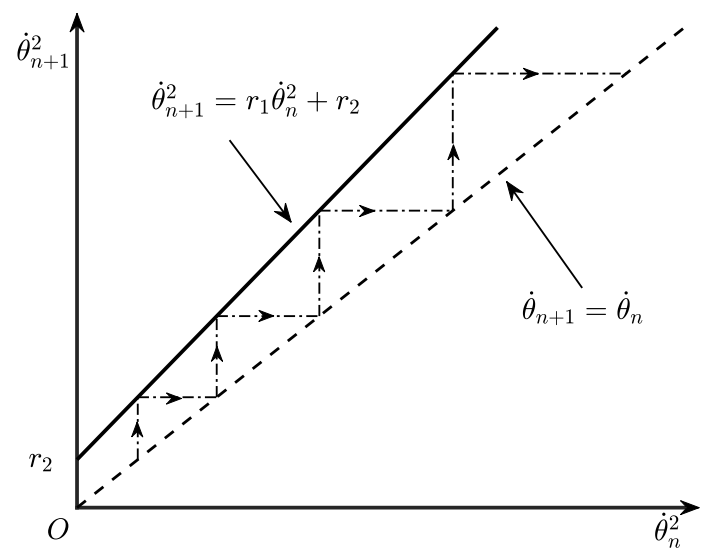

(b)

Fig. 3 Impact mapping Eq. (42) for (a) $q>1-1 / a$ and (b) $q<1-1 / a$.

we should have $B_{n}\left(\theta_{e}\right)=B_{n+1}\left(\theta_{e}\right)=\cdots=b_{e}$, and $\dot{\theta}_{n}^{+}=\dot{\theta}_{n+1}^{+}=\cdots=\dot{\theta}_{*}^{+}$. In this case, the mechanical energy residing in the system is given by,

$\widetilde{E}_{n}^{0}=\frac{1}{2} I_{1} a q_{c}^{n-1}\left(\dot{\theta}_{*}^{+}\right)^{2}+\frac{1}{2} m_{1} g h b q_{c}^{n-1}$.

By setting $\widetilde{E}_{n}^{0}=\widetilde{E}_{n}$, we obtain

$I_{1} a\left(\dot{\theta}_{*}^{+}\right)^{2}+m_{1} g h b=m_{1} g h b_{e}$,

from which, with the help of Eqs.(43) and (44), the critical mass exponent that could stop the wave propagation can be obtained.

In summary, the wave propagation of the massvarying domino chain could exhibit different modes, depending on the mass exponent $q$ and domino's configuration. We classify these propagation modes as below.

(1) Mode-A: For $q<k$, the solitary mode cannot be formed in the domino system.

(2) Mode-B: For $k<q<(1-1 / a)$, an accelerating solitary mode can be formed, and the front speed increases with the wave propagation.

(3) Mode-C: For $(1-1 / a)<q<q_{c}$, a uniform solitary mode can be formed with a constant front speed.

(4) Mode-D: For $q>q_{c}$, the wave propagation will be stopped by a heavy domino.

\section{Numerical investigations}

In our previous study [17], we have validated the numerical model in Eq. (3) by comparing numerical results with the experimental results in 25]. This section will use this numerical model as a reference to validate the accuracy of the proposed simplified model in this paper. Furthermore, we will study how the exponent $q$ and the domino configuration affect the wave propagation.

In the following studies, all the physical quantities are given in dimensionless form, where a natural speed scale $\bar{v}=\sqrt{g h}$, a natural time scale $\bar{t}=\sqrt{h / g}$ and a natural energy scale $\bar{E}=m_{1} g h / 2$ will be used.

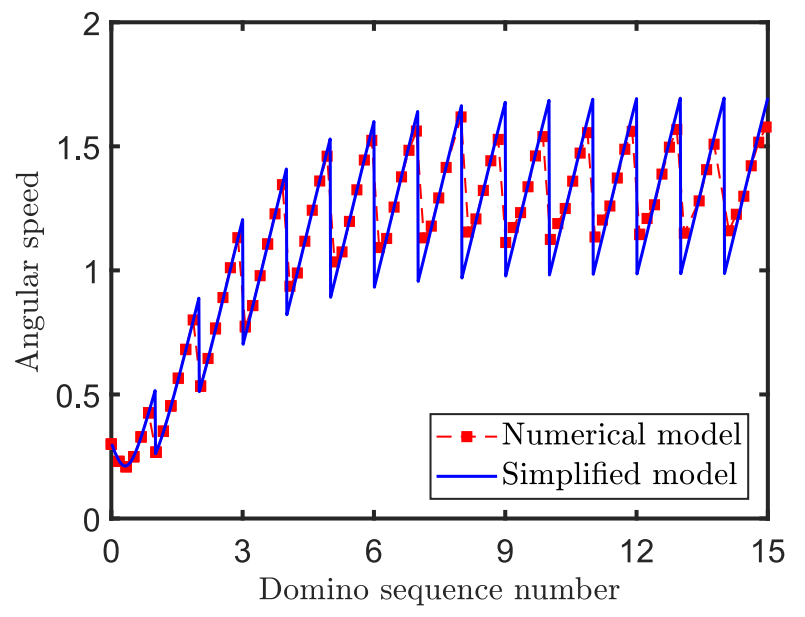

Fig. 4 Dimensionless angular speed of the front domino varies as a function of domino's sequence number.

\subsection{Validation of the simplified model}

In order to verify the validity of the simplified model, we firstly study a uniform domino system, i.e. $q=1$, which is the Domino $\mathrm{X}$ in [17] and was experimentally investigated in [25]. The thickness of the domino and the spacing between two neighboring dominoes are $\hat{d}=d / h=0.18$, and $\hat{s}=s / h=0.54$, respectively. In the simulations of the numerical model and the simplified model, the same initial condition $\dot{\theta}_{1}^{0}=$ $\dot{\theta}_{1}^{0} \bar{t}=0.3$ was used for the initial angular speed of the first domino. The other simulation parameters of 


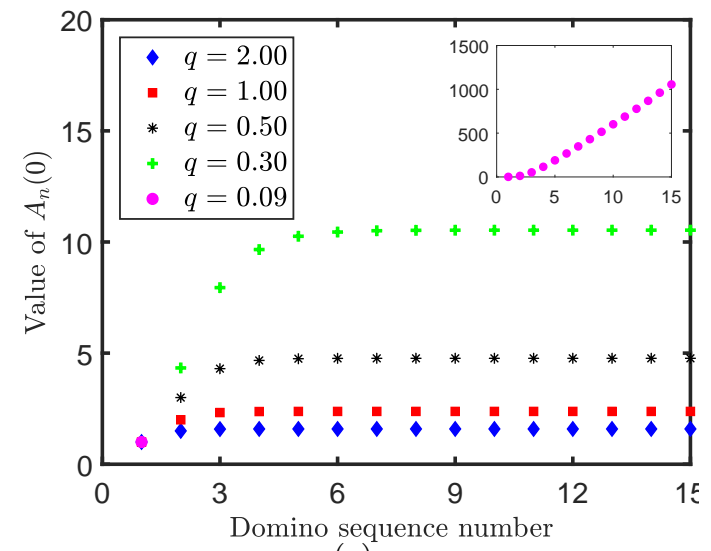

(a)

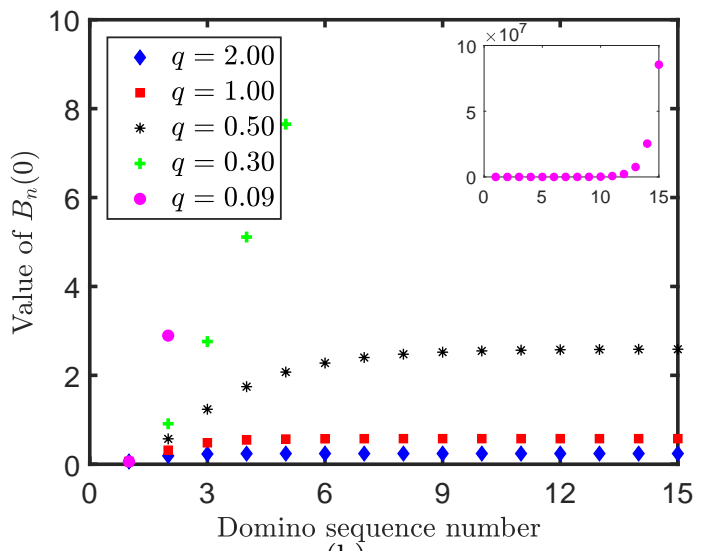

(b)

Fig. 5 (a) $A_{n}(0)$ and (b) $B_{n}(0)$ vary as functions of domino's sequence number and different exponential ratios calculated for $\hat{d}=0.15$ and $\hat{s}=0.45$. The results for $q=0.09$ are shown in the inset figures.

the numerical model include the friction coefficient between the dominoes $\mu_{1}=0$, the friction coefficient between the domino and the ground $\mu_{2}=0.9$, the restitution coefficient between the dominoes $e_{1}=0$, and the restitution coefficient between the domino and the ground $e_{2}=0$. For the simplified model, simulations of the impact-free and the impact phases were carried out by using Eqs. (19) and (28), respectively.

Fig. 4 shows the evolution of dimensionless angular speed of the front domino $\hat{\dot{\theta}}_{n}=\dot{\theta}_{n} \bar{t}$ for the numerical model (marked by red square-dashed line) and the simplified model (denoted by blue solid line). As can be seen from Fig. 4, a good agreement between the numerical model and the proposed simplified model has been achieved. Drastic variation of the angular velocities corresponds to the collision between the front domino and its next neighbouring domino. After some transient collisions, variation of the angular speed becomes stable, which indicates that the domino wave propagates in a uniform solitary mode with a constant natural speed (i.e. Mode-B).

\subsection{Front speed}

As it has validated that the simplified model is sufficient to describe the global behaviour of the domino system, we will use the simplified model to investigate the propagation speed of the mass-varying domino system. In our simulation, $\hat{d}=0.18, \hat{s}=0.54$, and the mass of the domino varies with its sequence number at the ratio $q$. Two initial conditions, $\hat{\dot{\theta}}_{1}^{0}=0.3$ and $\hat{\dot{\theta}}_{1}^{0}=2.0$, were used in our simulation for the initial rotational speed of the first topping domino corresponding to slow and fast toppling, respectively.
As $\hat{d}=0.18$ and $\hat{s}=0.54$, we obtain $k=0.302$ by using Eq. (13). According to Lemma 3 and Lemma 4, the convergence of $A_{n}$ and $B_{n}$ requires $q>k^{2}$ and $q>$ $k$, respectively. Fig. [5] demonstrates this convergence by showing the values of $A_{n}(0)$ and $B_{n}(0)$ calculated using Eqs. (32) and (34). For $q=0.5,1.0$ and $2.0>k$, it is obvious that both $A_{n}(0)$ and $B_{n}(0)$ converge to constant when $n>6$, and the constant decreases as $q$ increases. When $q=0.3<k, A_{n}(0)$ is convergent, and $B_{n}(0)$ is divergent. For $q=0.09<k^{2}$, it can be seen from the inner panels of Fig. 5 that both $A_{n}(0)$ and $B_{n}(0)$ are divergent.

Based on the time interval between the $n$th and the $(n+1)$ th collisions $t_{n}$, the transient propagation speed $v_{n}$ can be computed as $v_{n}=(s+d) / t_{n}$, and the dimensionless propagation speed can be calculated as $\hat{v}_{n}=v_{n} / \bar{v}$. Fig. 6(a) shows the dimensionless propagation speed of the domino system, $\hat{v}_{n}=v_{n} / \bar{v}$ as a function of domino's sequence number $n$. For $q=0.5,1.0$, and 2.0, we can calculate the limits of $A_{n}(0)$ as $a=4.77,2.37$ and 1.59 , respectively. So, the propagation speed for $q=0.5$ is divergent $(q<(1-$ $1 / a)$ ), and the ones for $q=1.0$ and 2.0 are convergent by using different initial speeds. For $q=1.0$ and 2.0, we can calculate the limits of $B_{n}(0)$ as $b=0.57$ and 0.23 , and therefore, the dimensionless natural speeds $\hat{v}^{*}=v^{*} / \bar{v}=1.69$ and 0.72 , respectively, by using Eq. (46). This result is consistent with the limits of $\hat{v}_{n}$ shown in Fig. 6(a) perfectly. The effect of domino's spacing on these speeds are shown in Fig. 6(b), which indicates that the natural speed decreases as domino's spacing increases for $q=1$, while the natural speed increases as domino's spacing increases when $q=2$. 


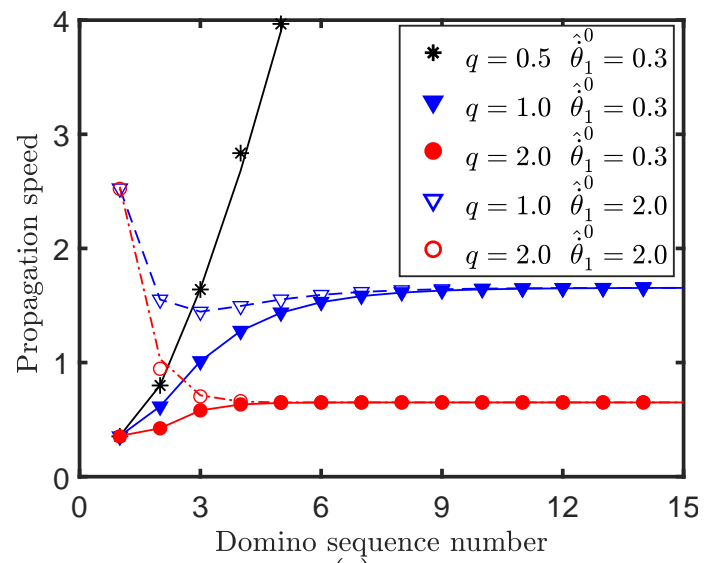

(a)

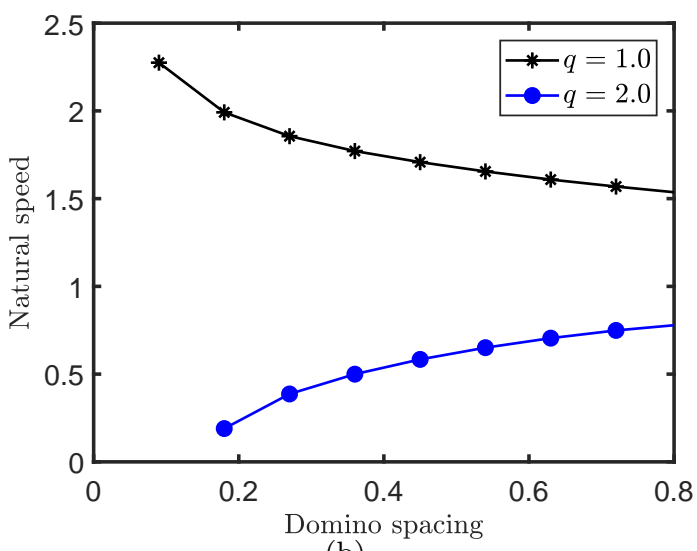

(b)

Fig. 6 (a) Dimensionless propagation speed $\hat{v}$ as a function of domino's sequence number calculated, and (b) natural speed $\hat{v}^{*}$ as a function of domino's spacing calculated for $\hat{d}=0.18$ and $\hat{s}=0.54$.

\subsection{Different propagation modes}

Wave propagation of the mass-varying domino system could exhibit different modes depending on the values of $q$ and $k$, which is determined by domino's geometry. Here, we fix $\hat{d}=0.18$, so $k$ can be considered as a function of domino's spacing $\hat{s}$. Once the values of $q$ and $k$ are known, the value of $a$ which is the limit of $A_{n}(0)$ under different $q$ and $\hat{s}$ can be calculated, and the value of $q_{c}$ can be obtained from Eq. (48). So, propagation mode can be determined based on the conditions given in Section 4.2. Fig. 7 presents the propagation mode of the domino system in the $q-\hat{s}$ plane, where the calculated results in Fig. 6 have been denoted in the figure. It can be seen that the domino systems with $q=1.0$ and 2.0 are in Mode-B, and the domino system with $q=0.5$ is in Mode-C.

Next, let us analyse the evolution of kinetic energy of the domino system. If the domino wave is in solitary modes, i.e. Mode-B or Mode-C, we can use Eqs. (17) and (32) to compute the kinetic energy of the domino system as

$T_{n}\left(\theta_{s}\right)=\frac{1}{2} I_{1} \dot{\theta}_{n}^{2} A_{n}\left(\theta_{s}\right) q^{n-1}$

According to Lemma 3, $A_{n}\left(\theta_{s}\right)$ for Mode-B and Mode$\mathrm{C}$ will converge to constant if $\mathrm{n}$ is sufficiently large. Therefore, the kinetic energy of the domino system at the instant when the $n$th domino just finishes collision can be expressed as

$T_{n}(0)=\frac{1}{2} I_{1} a q^{n-1}\left(\dot{\theta}_{n}^{+}\right)^{2}$

Fig. 8 shows the evolution of the dimensionless kinetic energy $\hat{T}_{n}(0)=T_{n}(0) /\left(1 / 2 m_{1} g h\right)$ and the ratio $R_{n}=T_{n+1}(0) / T_{n}(0)$ calculated using the simplified model for $q=2.0$ and 0.5 . It can be seen from the

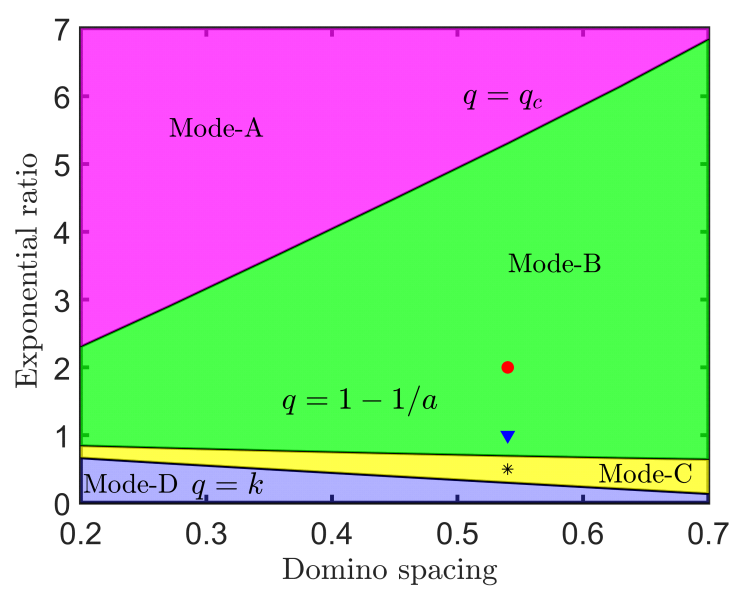

Fig. 7 Propagation modes of the domino system in the $q-\hat{s}$ plane. Propagation modes of the domino systems shown in Fig. [6] are marked as $\bullet, \nabla$ and $*$ for $q=2.0, q=1.0$ and $q=0.5$, respectively.

figure that the kinetic energy for $q=2.0$ increases exponentially with $n$, while for $q=0.5$, the kinetic energy does not vary monotonically. Our calculations show that $\lim _{n \rightarrow \infty} R_{n}=2$ for $q=2.0$, meaning that $R_{n}$ converges to $q$. However, $\lim _{n \rightarrow \infty} R_{n}=0.79$ for $q=0.5$, meaning that $R_{n} \neq q$. The reason is explained as follows. If the wave propagation is in Mode- $\mathrm{B}$, we have $\dot{\theta}_{n}^{+}=\dot{\theta}_{n+1}^{+}=\cdots=\dot{\theta}_{*}^{+}$, thus $T_{n}(0)=\frac{1}{2} I_{1} a\left(\dot{\theta}_{*}^{+}\right)^{2} q^{n-1}$, and the ratio $R_{n}=T_{n+1}(0) / T_{n}(0)$ will converge to $q$. If the wave propagation is in Mode-C, we have $\dot{\theta}_{n}^{+} \neq \dot{\theta}_{n+1}^{+}$from Eq. (42). In this mode, Eq. (42) can be simplified as $\left(\dot{\theta}_{n+1}^{+}\right)^{2} \approx r_{1}\left(\dot{\theta}_{n}^{+}\right)^{2}$, because the constant $r_{2}$ can be neglected when the value of $\dot{\theta}_{n}^{+}$is greatly enlarged for a large $n$. Therefore, the limit of $R_{n}$ in Mode-C can be expressed as $\lim _{n \rightarrow \infty} R_{n}=q r_{1}$. Note that $r_{1}=q(1-1 / a)=1.58$ for $q=0.5$, so we have 


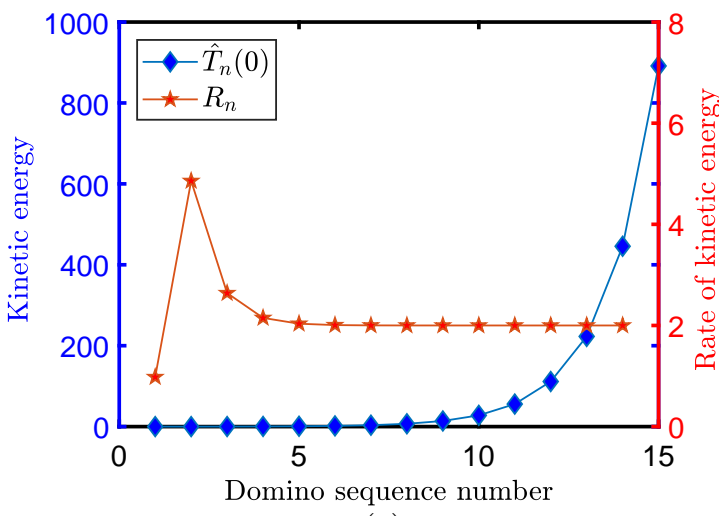

(a)

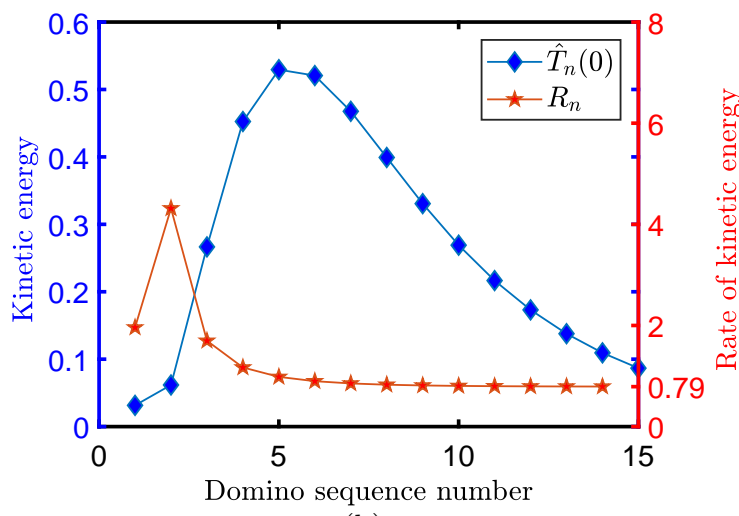

(b)

Fig. 8 Evolution of the dimensionless kinetic energy $\hat{T}_{n}(0)$ and the ratio $R_{n}=T_{n+1}(0) / T_{n}(0)$ for (a) $q=2.0$ and (b) $q=0.5$ calculated for $\hat{d}=0.18$ and $\hat{s}=0.54$.

$R_{n}=0.79$, which is consistent with the numerical results obtained by the simplified model.

\section{Concluding remarks}

This paper studied the toppling dynamics of a nonuniform domino system for which domino's mass varies exponentially with its sequence number. To analyse the characteristics of domino's wave propagation, we developed a simplified model whose configuration can be described by using the rotation angle of the front domino, and its equations of motion were built based on the conservation principle of mechanical energy and the principle of the generalized momentum conservation. The validity of the simplified model was tested by comparing with the numerical model studied in [17].

Based on the simplified model, we found that the coefficients of the kinetic and the potential energy can converge exponentially if the mass exponent $q$ is greater than the configuration-dependent parameter $k$. In this case, the domino propagates as a solitary wave at which the wavelength is a fixed number of dominoes. Furthermore, we obtained an analytical solution for domino's solitary wave by establishing an impact map for the evolution of its front velocity. It has been seen from the impact map that the front speed can converge to a fixed point if and only if the mass exponent $q$ is greater than a critical value. Therefore, the solitary wave can exhibit two different modes, which one is the uniform solitary mode for constant wave speed, and the other is the accelerating solitary mode for which the wave speed always accelerates. Furthermore, the kinetic energy of the domino system in the solitary mode will change exponentially with domino's sequence number.
If the mass exponent $q$ is very large, the domino wave can be blocked by a heavy domino after propagating a certain distance.

In conclusion, we have studied the dynamic characteristics and the energy evolution of the domino effect by using a mass-varying domino system. The results obtained from this study could benefit for understanding some intriguing phenomena in nature, and have potential engineering applications, such as design of the meta-material for energy transmission.

Acknowledgements This work has been supported by the National Natural Science Foundation of China under Grant No. 11702002, 11932001. The authors would like to specially thank Professor Jianhua Xie for providing inspirations.

\section{Compliance with ethical standards.}

Conflict of interest. The authors declare that they have no conflict of interest concerning the publication of this manuscript.

\section{References}

1. Ross $\mathrm{S}$ Stein. The role of stress transfer in earthquake occurrence. Nature, 402(6762):605, 1999.

2. Erik L Olson and Richard M Allen. The deterministic nature of earthquake rupture. Nature, 438(7065):212-215, 2005.

3. Andrew W Murray and Marc W Kirschner. Dominoes and clocks: the union of two views of the cell cycle. Science, 246(4930):614-621, 1989.

4. Tienchong Chang. Dominoes in carbon nanotubes. Physical review letters, 101(17):175501, 2008. 
5. Tienchong Chang and Zhengrong Guo. Temperature-induced reversible dominoes in carbon nanotubes. Nano letters, 10(9):3490-3493, 2010.

6. John M Kolinski, Pascale Aussillous, and L Mahadevan. Shape and motion of a ruck in a rug. Physical review letters, 103(17):174302, 2009.

7. Dominic Vella, Arezki Boudaoud, and Mokhtar Adda-Bedia. Statics and inertial dynamics of a ruck in a rug. Physical review letters, 103(17):174301, 2009.

8. Cyriel Wagemans. The nuclear fission process. CRC press, 1991.

9. Sevgi Aydin, Patricia M Friedrichsen, Yezdan Boz, and Deborah L Hanuscin. Examination of the topic-specific nature of pedagogical content knowledge in teaching electrochemical cells and nuclear reactions. Chemistry Education Research and Practice, 15(4):658-674, 2014.

10. Diego Martín-Cano, ML Nesterov, AI FernandezDominguez, FJ Garcia-Vidal, Luis Martin-Moreno, and Esteban Moreno. Domino plasmons for subwavelength terahertz circuitry. Optics Express, 18(2):754-764, 2010.

11. Sailing He, Yingran He, and Yi Jin. Revealing the truth about 'trapped rainbow'storage of light in metamaterials. Scientific reports, 2:583, 2012.

12. Bolei Deng, Pai Wang, Qi He, Vincent Tournat, and Katia Bertoldi. Metamaterials with amplitude gaps for elastic solitons. Nature communications, 9 (1):3410, 2018.

13. Jean-Philippe Boucher, Christophe Clanet, David Quéré, and Frédéric Chevy. Popsicle-stick cobra wave. Physical review letters, 119(8):084301, 2017.

14. Xiaoyan $\mathrm{Lu}$, Zuhuang Chen, Ye Cao, Yunlong Tang, Ruijuan Xu, Sahar Saremi, Zhan Zhang, Lu You, Yongqi Dong, Sujit Das, et al. Mechanical-force-induced non-local collective ferroelastic switching in epitaxial lead-titanate thin films. Nature communications, 10(1):1-8, 2019.

15. Matty Demont, Wim Daems, Koen Dillen, Erik Mathijs, Christophe Sausse, and Eric Tollens. Regulating coexistence in europe: Beware of the domino-effect! Ecological economics, 64(4):683$689,2008$.

16. James S Olson and Randy W Roberts. Where the Domino Fell: America and Vietnam 1945-1995. John Wiley \& Sons, 2011.

17. Tengfei Shi, Yang Liu, Nannan Wang, and Caishan Liu. Toppling dynamics of regularly spaced dominoes in an array. Journal of Applied Mechanics, 85(4):041008, 2018.
18. DE Daykin. Falling dominoes. SIAM Review, 13 (4):569, 1971.

19. DE Shaw. Mechanics of a chain of dominoes. American Journal of Physics, 46(6):640-642, 1978.

20. BG McLachlan, G Beaupre, AB Cox, and L Gore. Falling dominoes (de daykin). SIAM Review, 25(3): 403, 1983.

21. Charles W Bert. Falling dominoes. SIAM Review, 28(2):219-224, 1986.

22. Costas J Efthimiou and Michael D Johnson. Domino waves. SIAM review, 49(1):111-120, 2007.

23. Ron Larham. Validation of a model of the domino effect? arXiv preprint arXiv:0803.2898, 2008.

24. Wilam J Stronge. The domino effect: a wave of destabilizing collisions in a periodic array. Proceedings of the Royal Society A: Mathematical, Physical and Engineering Science, 409(1836):199208, 1987.

25. W J Stronge and D Shu. The domino effect: successive destabilization by cooperative neighbours. Proceedings of the Royal Society A: Mathematical, Physical and Engineering Science, 418(1854):155$163,1988$.

26. J M J Van Leeuwen. The domino effect. American Journal of Physics, 78(7):721-727, 2010.

27. Fumio Fujii, Yoshihiro Inoue, and Takahiro Nitta. Modeling the domino wave propagation in contact mechanics. Transactions of The Japan Society of Mechanical Engineers Series C, 78(788):1133-1142, 2012.

28. Guang Lu, James $\mathrm{R}$ Third, and Christoph $\mathrm{R}$ Müller. Effect of particle shape on domino wave propagation: a perspective from $3 \mathrm{~d}$, anisotropic discrete element simulations. Granular matter, 16 (1):107-114, 2014.

29. Caishan Liu, Zhen Zhao, and Bernard Brogliato. Frictionless multiple impacts in multibody systems. i. theoretical framework. Proceedings of the Royal Society of London A: Mathematical, Physical and Engineering Sciences, 464(2100):3193-3211, 2008.

30. Caishan Liu, Zhen Zhao, and Bernard Brogliato. Frictionless multiple impacts in multibody systems. ii. numerical algorithm and simulation results. Proceedings of the Royal Society of London A: Mathematical, Physical and Engineering Sciences, 465(2101):1-23, 2009.

31. Bernard Brogliato. Nonsmooth mechanics. Springer, London, 1999.

32. Caishan Liu, Hongjian Zhang, Zhen Zhao, and Bernard Brogliato. Impact-contact dynamics in a disc-ball system. Proceedings of the Royal Society A: Mathematical, Physical and Engineering Science, 469(2152):20120741, 2013. 
33. Jiao Wang, Caishan Liu, and Zhen Zhao. Nonsmooth dynamics of a $3 \mathrm{~d}$ rigid body on a vibrating plate. Multibody System Dynamics, 32(2):217-239, 2014.

34. Wayne H Enright, TE Hull, and Bengt Lindberg. Comparing numerical methods for stiff systems of ode: s. BIT Numerical Mathematics, 15(1):10-48, 1975.

35. Kenneth Langstreth Johnson and Kenneth Langstreth Johnson. Contact mechanics. Cambridge university press, 1987.

36. Daolin Ma and Caishan Liu. Contact law and coefficient of restitution in elastoplastic spheres. Journal of Applied Mechanics, 82(12):121006, 2015. 